\title{
Les effets de la politique du logement sur l'évolution du taux de propriétaires en France
}

\section{Catherine Bonvalet et Arnaud Bringé}

\section{OpenEdition}

\section{Journals}

Édition électronique

URL : http://journals.openedition.org/ress/2342

DOI : $10.4000 /$ ress. 2342

ISSN : $1663-4446$

Éditeur

Librairie Droz

Édition imprimée

Date de publication : 1 juin 2013

Pagination : 153-177

ISBN : 978-2-600-01749-7

ISSN : 0048-8046

Référence électronique

Catherine Bonvalet et Arnaud Bringé, « Les effets de la politique du logement sur l'évolution du taux de propriétaires en France », Revue européenne des sciences sociales [En ligne], 51-1 | 2013, mis en ligne le

01 janvier 2017, consulté le 19 avril 2019. URL : http://journals.openedition.org/ress/2342 ; DOI :

$10.4000 /$ ress.2342 


\title{
LES EFFETS DE LA POLITIQUE DU LOGEMENT SUR L'ÉVOLUTION DU TAUX DE PROPRIÉTAIRES EN FRANCE
}

CATHERINE BONVALET \& ARNAUD BRINGÉ

INED, Paris

bonvalet@ined.fr/bringe@ined.fr

Résumé. Cet article se propose d'étudier les effets des politiques du logement menées en France depuis 1973 sur les taux de propriétaires au sein des différentes générations. Les réformes du crédit bancaire, les lois favorisant l'accession à la propriété, en particulier la loi de 1977, ont surtout profité aux générations du baby-boom. Cependant toutes les classes sociales et les types de ménages n'ont pas bénéficié de la même manière des réformes, l'écart se creusant entre les classes supérieures ou moyennes et les ouvriers ou employés, entre les couples et les femmes avec enfants. Par ailleurs, les évolutions de la famille avec l'augmentation des divorces et séparations ont généré de nouveaux parcours résidentiels initiés par les premières générations du baby-boom avec un retour vers le locatif après une accession à la propriété. Elles expliquent en partie le ralentissement de la hausse des taux de propriétaires au cours des deux dernières décennies.

Mots-clés: politique du logement, propriété, génération, inégalités sociales, famille.

\begin{abstract}
This article examines the effect of French housing policy since 1973 on home ownership rates across different birth cohorts. The baby-boom generations profited most from banking reforms that gave wider access to credit and new laws favouring home ownership, notably that of 1977. However, not all social classes and types of households have benefited equally from these reforms. A widening gap has emerged between the upper and middle classes, on the one hand, and manual and clerical workers, on the other; and also between couples and single mothers. At the same time, the modifications of family patterns, with the rise in divorce and separation, have led to new residential trajectories initiated by the first baby-boom generations, with a return to the rental market after a period of home ownership. This partly explains the slower increase in home ownership rates over the last two decades.
\end{abstract}

Keywords: housing policy, ownership, generation, social inegality, family. 
En 50 ans, la France est devenue un pays de propriétaires où près de $60 \%$ possède son logement contre $35 \%$ en 1954. Grâce au développement du crédit et aux politiques de logement, l'achat de la résidence principale n'est plus «un rêve», mais une réalité qui s'offre à la majorité des ménages. Le recours à l'emprunt qui s'est opéré au cours de ces dernières décennies a entraîné une «révolution urbaine» qui a concerné toutes les couches de la société. Anne Gotman l'a décrite en ces termes :

C’est aux alentours des années 1930 que la part relative des avoirs immobiliers redevient majoritaire dans la structure du patrimoine, après avoir décliné rapidement durant la seconde moitié du $\mathrm{XIX}^{\mathrm{e}}$ siècle. Il faut toutefois attendre les années 1950, c'est-à-dire une bonne expérience de l'érosion monétaire, pour que le mythe de la pierre fasse de l'immobilier le placement populaire par excellence. Entre-temps, le rentier s'est discrètement retiré du paysage social (Gotman, 1986, p. 35)

Ce qui était utopie au XIX siècle, demi-échec entre les deux guerres, se concrétise enfin au cours de la seconde moitié de notre siècle (Groux et Levy, 1993).

Le crédit a bouleversé le calendrier de la propriété qui n’est plus le fruit d’une épargne ou le résultat d'un héritage. Désormais le choix entre la location et la propriété du logement constitue un événement majeur du cycle de vie et structure fortement les trajectoires résidentielles. Il intervient très tôt au moment même où se constitue la famille. Pierre Bourdieu avait montré comment l'arrivée des enfants et l'accession à la propriété allaient de pair.

La question de l'achat d'une maison se pose avec une force particulière à certaines étapes du cycle de vie, en relation avec le souci de fonder une famille, c'est-à-dire au moment du mariage ou dans les années qui suivent, en liaison avec l'apparition des enfants (Bourdieu, 2000, p. 50).

Statistiquement les liens entre famille et propriété se vérifient: les couples propriétaires ont une fécondité plus élevée que les locataires (Bonvalet, 1978), les couples mariés sont plus propriétaires que les cohabitants (Sarma, 1985) ou que les personnes seules avec ou sans enfant. Les politiques du logement ont accompagné, voire encouragé, ce désir de stabilité des ménages. Cette priorité 
donnée à l'accession à la propriété depuis la fin du XIX ${ }^{\mathrm{e}}$ siècle n'est toujours pas exempte d'idéologie comme l'écrit Jean-Claude Driant (2009).

En réalité, la relation «famille nucléaire / accession à la propriété » est l’avatar moderne du couple famille / propriété dont on retrouve la trace au cours des siècles passés; et les phénomènes contemporains d’accession à la propriété prennent leur véritable dimension si l'on porte sur eux un regard historique. Dans notre société, la propriété est investie d'une forte valeur symbolique et idéologique que la Révolution Française a contribué à renforcer à travers une certaine cristallisation des sentiments qu'inspirent la famille et le patrimoine. Le complexe «famille / propriété » va fournir les bases de tout un courant de pensée du XIX siècle dont Bonald et Le Play, d'une manière différente, sont les représentants. Au XIX et au début du XX siècle, la propriété familiale de la maison individuelle apparaît comme la solution idéale aux problèmes de logement engendrés par l'industrialisation et l'urbanisation. Elle devait également contribuer à «construire la famille ». Il s'agissait en fait de domestiquer la famille à travers la propriété (Joseph, 1976).

Depuis leur naissance à la fin du XIX siècle, les politiques du logement ont toujours cherché à encourager la propriété occupante, cet objectif est devenu encore plus visible depuis la réforme de 1977 qui tend de faire de la propriété «l'aboutissement logique» de tout parcours résidentiel ascendant (Driant, 2009).' Au-delà du souci de répondre aux aspirations des ménages², existent d’autres préoccupations : d'une part la propriété est toujours envisagée comme un élément de stabilité sociale, d'autre part l'accession à la propriété est un moyen de soutenir l'activité du bâtiment et les aides à l'accession à la propriété sont moins onéreuses que les aides destinées au locatif, les accédants prenant

I Cependant, contrairement à d'autres pays européens dont les politiques se sont presque exclusivement centrées sur la propriété (Louvot, 200I; Withehead, 2009; Bugeja, 20II), la France a réussi à maintenir un équilibre entre les différents statuts d'occupation, notamment en gardant un secteur locatif social.

2 Une des premières enquêtes de l'INED, «Désirs des Français en matière d'habitation urbaine» de 1945, réalisée par Alain Girard, a mis en évidence le désir de propriété des ménages (64\%) et leur souhait d'habiter une maison individuelle (72\%). Ce dernier écrit «notre avenir démographique est en partie solidaire d'une politique nationale de l'habitat». 
en charge une partie du coût du logement (Bosvieux, 2005). Ce sont les raisons pour lesquelles les politiques du logement ont toujours cherché, de manière plus ou moins forte selon la couleur politique des gouvernements successifs, à stimuler l'épargne des ménages par l'achat du logement (Driant, 2009).

Selon la période où ils se présenteront sur le marché du logement, en fonction de la politique du moment, du contexte économique favorable ou non à l'accession à la propriété, les ménages n’auront pas les mêmes opportunités pour devenir propriétaires. Dans cet article, on se propose d'étudier à partir des enquêtes Insee Logement de 1978 à 2006, les effets des politiques d'accession à la propriété sur les taux de propriétaires au sein des différentes générations.

\section{QUELQUES ÉLÉMENTS DE POLITIQUE DU LOGEMENT}

Si la question du logement en Europe a été au centre des réflexions des réformateurs sociaux du XIX ${ }^{\mathrm{e}}$ siècle, l'intervention de l'État ne deviendra réellement légitime qu’après la Seconde Guerre mondiale en raison de la crise du logement (Mouillart, 1992). Le même constat peut être dressé pour la France, même si de 1850 à 1950 les bases qui constitueront l'armature du logement social et de l'aide à la pierre sont progressivement posées (Driant, 2009). Malgré les graves problèmes de logement que rencontrent les classes populaires, les interventions publiques restent modestes. Il fallut la crise de logement d’aprèsguerre pour que l’État se décide à mener une véritable politique du logement. En effet, malgré la situation catastrophique du parc de logements, l'effort massif de construction ne débutera réellement qu’à partir du milieu des années 1950 avec la mise en chantiers des grands ensembles, le secteur HLM assurant dès I957 près de $30 \%$ de la construction neuve (ibid.) d'une part et la réforme du crédit d'autre part avec les HLM-accession et surtout les prêts du Crédit foncier, notamment les «logecos» (1953) (Merlin, 1988). Mais cette diffusion de la propriété, dont Christian Topalov (1987) a retracé l'histoire, ne s'est pas faite de manière linéaire. La période 1955-1963 se caractérise par une forte croissance de la production de logements neufs, en particulier de logements destinés à la propriété. De nombreux cadres supérieurs et moyens résolvent leurs problèmes de logement en achetant leur résidence principale. Cependant, les 
types de logement mis sur le marché et les prêts qui les accompagnent (notamment ceux du Crédit Foncier) favorisent également l'augmentation du nombre de propriétaires parmi les ouvriers et les employés. Cette période est suivie, de 1963 à 1968, d’un fléchissement qui touche toutes les catégories sociales, spécialement les ouvriers. Ensuite, la diffusion de la propriété reprend, mais à un rythme moins rapide que celui des années 1950.

Figure I. Évolution de la part des propriétaires occupants dans l'ensemble des résidences principales

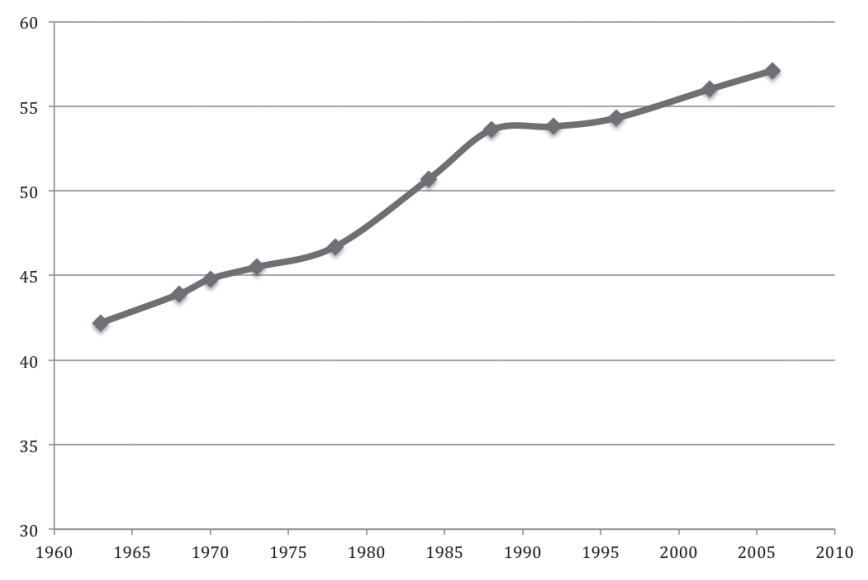

Source: Enquêtes Logement INSEE

Grâce à cette politique publique, les jeunes ménages confrontés à la crise du logement accèderont au confort en entrant dans un HLM situé dans un grand ensemble ou accédant à la propriété dans de nouveaux lotissements. En 1965, la création de l'épargne logement puis la création en 1967 d'un marché hypothécaire faciliteront encore plus le recours à l'emprunt si bien quavec la hausse des loyers (augmentation de II \% entre 1953 et 1962 contre $4 \%$ pour les prix) l'écart entre un loyer libre et une mensualité d'emprunt a été considérablement réduit. Si le pavillon du périurbain encouragé par les gouvernements, notamment par Chalendon de 1969 à 1972, devient l'habitat souhaité par les Français³, les grands ensembles en revanche font l'objet de nombreuses critiques. Plusieurs rapports 
(Livre Blanc HLM, 1975 ; rapport Barre, 1976) estiment que la crise du logement est désormais résorbée et qu'il n’est plus nécessaire de soutenir l'activité de la construction neuve, l'essentiel étant de donner aux ménages la liberté de choisir leur statut d'occupation et leur type d'habitat en passant de l'aide à la pierre à l'aide à la personne. La réforme du logement de 1977, en créant le PAP (prêt aidé à l'accession à la propriété) et l'APL (aide personnalisée au logement) avait pour objectif d’aider les ménages à assumer le coût du logement notamment en facilitant l'accession dans le neuf. La réforme du logement de 1977 entraîne la mise sur le marché de nouveaux produits: la fabrication industrielle de pavillons attire une nouvelle clientèle recrutée, dans la grande majorité, parmi les ouvriers qualifiés, les employés et les cadres moyens. Grâce à ces politiques favorisant l'accession à la propriété, le taux de propriétaires est passé de $45 \%$ en 1973 à près de $54 \%$ en I988. Mais la crise économique, la baisse rapide de l'inflation, les contraintes budgétaires des années 1980 ont mis en évidence les limites de la réforme de 1977 et ont conduit les gouvernements à réduire les aides aux logements puis à la réviser en 1995 avec la création des prêts à taux zéro réservés aux primo-accédants. Ces dernières mesures ont permis, avec la chute des taux d'intérêts et l'allongement de la durée des prêts, une reprise de l'accession à la propriété à la fin des années 1990. Cette reprise s’effectue dans un contexte économique porteur: le niveau de vie moyen des Français progresse de ro\% hors inflation entre 1996 et 200 (Bosvieux, 2005). Toutefois, la croissance du taux de propriétaires ne retrouvera pas le niveau des années 1980. En 2006, le taux de propriétaire atteindra $57 \%$ (Figure I).

\section{2. ÉVOLUTION DU TAUX DE PROPRIÉTAIRES PAR ÂGE ET PAR GÉNÉRATION}

Entre 1978 et 1988, la diffusion de la propriété est particulièrement sensible. À tous les âges pratiquement, on observe entre l'enquête logement de 1978 et celle de 1988 une augmentation de la proportion de propriétaires, à l'exception des moins de 30 ans et des plus de 75 ans. La grande majorité des générations profiteront de cet effet du moment mais de manière plus ou moins intense. Cette hausse des taux est particulièrement forte entre 30 et 65 ans. Cependant, après 50 ans, il ne semble pas que l'on puisse imputer cette augmentation unique- 
ment à la réforme de 1977. En effet, la figure 2 met nettement en évidence une forte progression de la proportion de propriétaires parmi les 60-65 ans qui provient plus d'un effet de génération que d'un effet de période. En 1978, les personnes de cet âge appartiennent aux générations nées durant le premier conflit mondial qui, déjà insérées sur le marché immobilier, souvent dans des logements dont les loyers avaient été bloqués, n’ont pas profité de la politique du logement des années 1950. Dix ans plus tard, en I988, arrivent à cet âge les générations nées de I924 à I928 qui vont connaître, jeunes adultes, toutes les transformations des modes de financement du logement favorisant l'accession à la propriété ainsi que la relance de la construction neuve d’après-guerre.

Figure 2. Évolution entre 1973 et 2006 de la proportion de ménages propriétaires de leur logement en fonction de l'âge

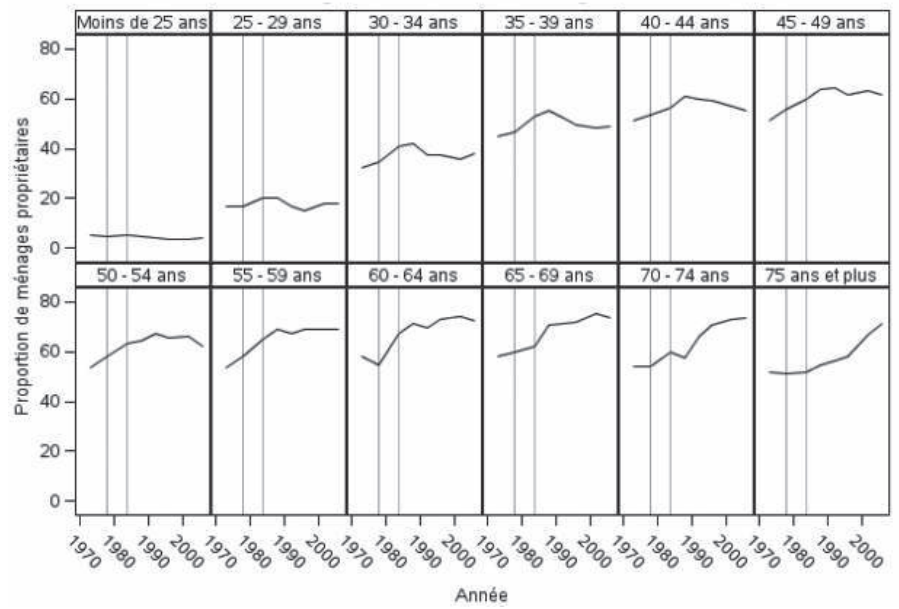

Source: Enquêtes Logement INSEE 
À partir des années cinquante, comme on l’a vu, se produit une révolution dans le financement du logement avec le recours à l'emprunt et les ménages, qui au départ étaient réticents, se laissent d'autant plus séduire par ce mode d'achat que la loi de 1948 met fin au blocage des loyers dans les logements construits à partir du $\mathrm{I}^{\mathrm{er}}$ janvier $1949 .{ }^{4}$ Mais pour cela, il a fallu que s'effectue dans cette génération «une acculturation par le crédit». À cette époque, l’endettement suscite encore des craintes et fait l'objet d'une certaine répugnance héritée des siècles passés. Avec la banalisation des crédits, notamment avec l'achat à crédit d'une automobile, les défiances à l'égard de l'endettement s'estompent et petit à petit, le nombre d’accédants dépasse celui des propriétaires. Le recours au crédit devient alors, au cours de cette période, la principale voie pour accéder au statut de propriétaire. Et les générations nées entre les deux guerres seront les premières à initier ce mode d’accès à la propriété.

$\mathrm{Au}$ début des années 1960, période à laquelle démarre réellement la construction des grands ensembles et des logements destinés à la propriété, les personnes nées entre 1925 et 1940 ont entre 25 et 35 ans. C'est l'âge où se pose la question du choix du logement, plus précisément du choix du statut d'occupation, en liaison avec la constitution de la famille. A la fin des années 1970, les générations nées après guerre vont à leur tour commencer leur parcours logement et pourront alors pleinement bénéficier de la réforme du logement de 1977 notamment en achetant leur logement grâce au prêt PAP et à l'APL.

4 Les loyers anciens triplent en 6 ans et les loyers des logements neufs deviennent libres II faut rappeler que les Parisiens, après plusieurs décennies de blocage de loyer dû au moratoire de 1914, n'étaient pas habitués à payer des loyers élevés. Leurs loyers ne dépassaient pas 10\% de leurs revenus alors que généralement les loyers représentent entre 25 et $35 \%$ des revenus, (voir Sauvy, 1965). 
Figure 3. Proportion de propriétaires à différents âges

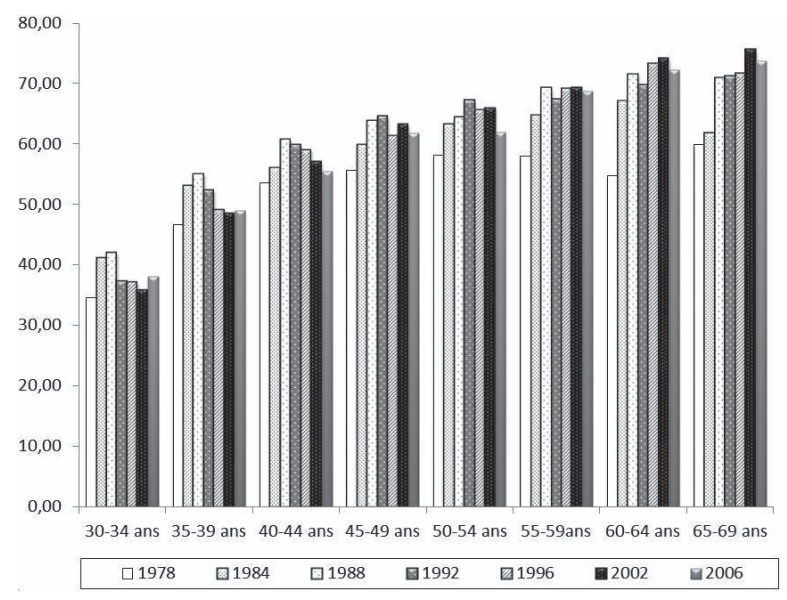

Source: Enquêtes Logement INSEE

Une autre manière de mettre en évidence les effets des politiques consiste à étudier de manière fine au cours du temps l'évolution des taux de propriétaires aux différents âges. Si l’on considère les ménages dont la personne de référence est âgée de 35 à 39 ans, c'est-à-dire les ménages qui se trouvent au moment du cycle de vie où s'opèrent les choix stratégiques en matière familiale et résidentielle, on observe que la proportion de propriétaires passe de 46,67\% en 1978 à 55,06\% en 1988 pour diminuer ensuite et se stabiliser à 49,00\% en 2006. Les générations nées entre 1949 et 1953 apparaissent donc en meilleure position que les générations qui les précèdent et qui les suivent. Aux âges de 45-49 ans, il semble que ce soient les ménages en 1992 qui atteignent le plus haut taux de propriétaires avec près de $65 \%$ contre $57 \%$ en 1978 et $62 \%$ en 2006. À la veille de la retraite, avant 60 ans, les ménages appartenant aux générations nées entre 1929 et I933 sont plus nombreux à détenir leur résidence principale. En fait, hormis les plus âgés nés avant 1923 dont le taux de propriétaires s'élève à $58 \%$ en 1978 et ceux nées entre 1925 et 1929 (65\%), les différences restent faibles, un peu en dessous des $70 \%$ de propriétaires. Après 65 ans, l'effet de génération et de période apparaît clairement et confirme que les générations nées avant 1920 n’ont pas pu bénéficier du développement du crédit alors 
que les jeunes ménages nés dans l'entre deux guerres se présenteront sur le marché du logement au moment même où se met en place la réforme du crédit (Topalov, 1987). Certains d'entre eux ont effectué un passage par le logement social et profité des mesures des années 1960-1970 pour réaliser un parcours résidentiel ascendant (Bonvalet et Bringé, 1988; Levy, 2007; Arrondel, 2007).

À partir des enquêtes Logement, il a été possible de reconstituer l'évolution des taux de propriétaires par génération de manière à situer les générations du baby-boom. En effet, ces dernières ont fait l'objet de nombreux débats quant à savoir si elles étaient privilégiées, dorées, bénies (Chauvel, I998 ; Bonvalet et al 20I I ; Clerc et al., 20I I). Par rapport à leurs aînés, les ménages dont la personne de référence appartient aux premiers nés du baby-boom (1946-1950) ne détiennent pas plus, en fin de compte, leur résidence principale comme le montre la figure 4. En revanche, on observe bien une diffusion plus rapide de la propriété aux âges de 30 à 40 ans pour cette génération que pour les générations précédentes. Les baby-boomers arrivent à cette étape du cycle de vie entre 1976 et 1990, c'est-à-dire au moment où se produisent tous les effets de la réforme du logement de 1977. Par ailleurs, le contexte leur est particulièrement favorable avec des taux d'intérêt réels très faibles en raison de l'inflation et de la déduction des intérêts d'emprunt du revenu imposable.

Figures 4 et 5 . Évolution en fonction de l'âge de la proportion de ménages propriétaires de leur logement par génération
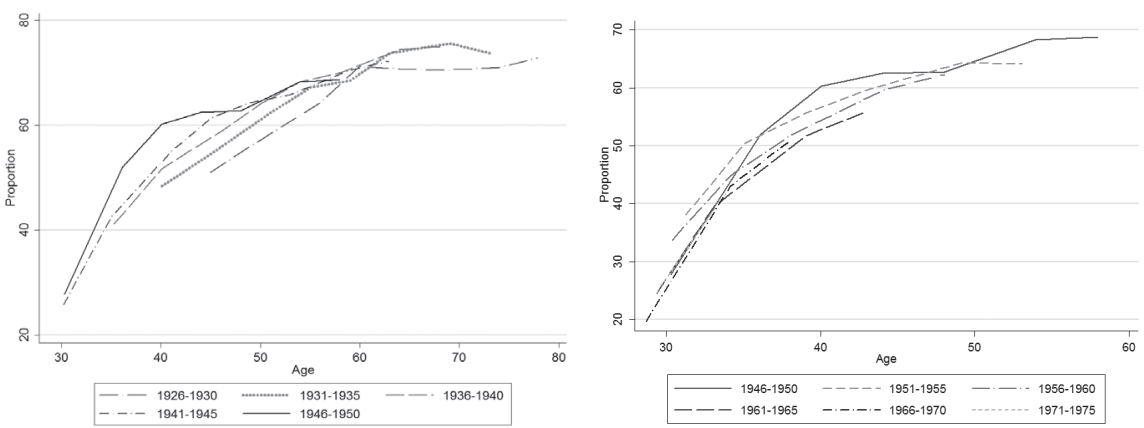
Comme le souligne Anne Laferrère, la tendance s'est inversée pour les générations suivantes, l'inflation est maîtrisée, les taux d’intérêts réels sont positifs, les intérêts d'emprunt immobiliers ne sont plus déductibles (Laferrère, 2007) et les aides publiques moins généreuses. Les générations nées après 1950 connaissent donc une conjoncture nettement moins favorable après 35 ans. En début de cycle de vie, elles bénéficient encore des effets de la loi de 1977 et des aubaines de la conjoncture des années I980; la proportion de ménages propriétaires est supérieure à celle des baby-boomers avant 35 ans. En revanche, entre 35 et 49 ans, la proportion de propriétaires recule (Figure 5) et l'âge médian d'accès à la propriété après s'être stabilisé repart à la hausse (Clerc et al, 20 I). En résumé, il semble bien que les générations nées tout de suite après la guerre aient connu des conditions assez exceptionnelles qui ont profondément modifié leur calendrier d'accession à la propriété. Elles leur ont permis d’acquérir plus jeunes leur résidence principale et parfois de renouveler cette opération quelques années plus tard. Cependant cet avantage ne s'est pas poursuivi tout au long de cycle de vie puisqu'à 60 ans les ménages ${ }^{5}$ dont la personne de référence appartient aux premières générations du baby-boom, donc née entre I946 et I950, ne détiennent pas plus leur logement à la veille de la retraite. D’autres facteurs sont intervenus : d’une part avec l’augmentation de l'espérance de vie, l'héritage intervient de plus en plus tardivement dans le cycle de vie, d'autre part, les parcours sont devenus plus complexes avec l'augmentation des divorces et la montée du chômage après 50 ans.

5 Signalons que cette analyse est réalisée à partir des ménages enquêtés lors des enquêtes Logement. Les résultats diffèrent lorsque l'on considère non plus les ménages mais les individus (voir l'étude de Clerc, Monso, Pouliquen sur les inégalités entre les générations depuis le baby-boom, 2011). 


\section{3. ÉVOLUTION DU TAUX DE PROPRIÉTAIRES PAR ÂGE ET TYPE DE MÉNAGE}

Les politiques du logement ont toujours eu pour cible privilégiée les familles. $\mathrm{Au} \mathrm{XIX}^{\mathrm{e}}$ siècle, il s'agissait de sortir les familles des taudis et des logements insalubres où elles s'entassaient. Après la Seconde Guerre mondiale, la pénurie de logements liée au manque de construction de l'Entre-deux-guerres et aux démolitions de la guerre 1940 est d'autant plus grande que de nouveaux besoins de logement émergent avec le baby-boom. Il fallait construire vite et beaucoup pour donner un logement aux jeunes ménages et aux migrants venus des campagnes et de l'étranger. Ainsi, en même temps que se consolidait l'idée d'un modèle familial unique - le couple avec enfants conforté par la reprise de la natalité et le rajeunissement de l'âge au mariage -, apparaissaient dans le paysage urbain les grands ensembles, puis les lotissements de maisons individuelles. Petit à petit une famille type a correspondu un logement type : la maison en accession à la propriété. En 1978, 55\% des couples mariés avec enfants dont la personne de référence était âgée entre 30 et 40 ans habitait une maison individuelle et $48 \%$ étaient propriétaires, près de 30 ans plus tard ces proportions s'élèvent respectivement à 7i \% et $66 \%$ (Arbonville, Bonvalet, 2006).

Figure 6. Proportion de propriétaires selon le type des ménages dont la personne de référence est âgée de 35 à 39 ans

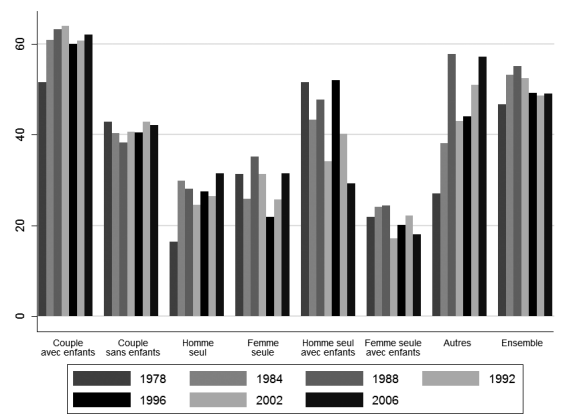

Figure 7. Proportion de propriétaires selon le type des ménages dont la personne de référence est âgée de 60 à 64 ans

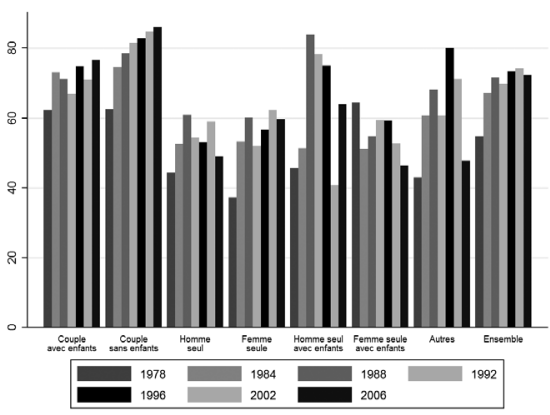


Comme de nombreuses recherches l’ont montré (Bonvalet et Merlin, I988 ; Cuturello, 1993), la propriété demeure avant tout une affaire de couple, surtout en milieu de cycle de vie. Les figures 6 et 7 confirment l'analyse précédente, à savoir que ce sont bien les couples avec enfants appartenant aux générations nées entre 1945 et 1957 qui ont bénéficié d'une conjoncture favorable pour devenir propriétaires. Au moment de la retraite aux âges de 60-64 ans, la proportion de propriétaires la plus forte s'observe parmi les couples sans enfant (Figure 5). Depuis 40 ans, cette proportion a connu une hausse continue passant de $62 \%$ en 1978 à $86 \%$ en 2006.

Ces quelques chiffres montrent l'impact des politiques du logement sur les modes de vie des familles. Cependant dès le milieu des années ı980, plusieurs auteurs ont émis des réserves à l'égard d'une politique axée uniquement sur l'accession à la propriété (Bonvalet et Merlin, I988 ; Arbonville, 2006 ; CAS, 20Io). En effet, si la maison individuelle répond aux aspirations des jeunes couples avec enfants, elle ne correspond pas aux souhaits des autres ménages qui recherchent la location, la centralité et l'accessibilité.

Or si la vie familiale se déroulait dans les années 1950 de façon linéaire (mariage, naissance des enfants, décohabitation, veuvage), elle est devenue une succession de séquences où alternent vie en couple et vie solitaire avec ou sans enfant.

Figure 8. Répartition des hommes âgés de 60 ans selon la génération

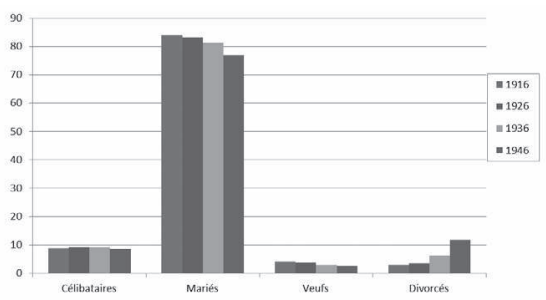

Figure 9. Répartition des femmes âgées de 60 ans selon la génération

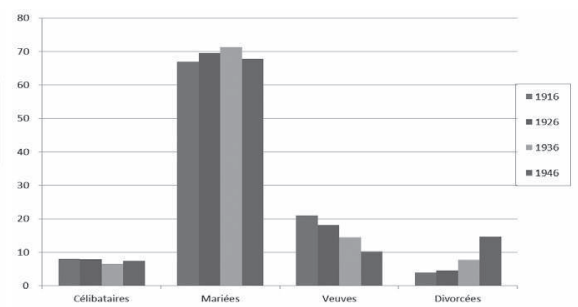

Source: Enquêtes Logement INSEE 
La complexité des trajectoires familiales apparaît clairement dans les changements de la répartition des hommes et des femmes à 60 ans selon l'état matrimonial des différentes générations. Si la proportion de femmes mariées évolue peu, en revanche le poids des femmes divorcées augmente considérablement passant de $5 \%$ dans la génération 1926 à I $5 \%$ dans la génération 1946, compensant ainsi le recul de la mortalité (la part des veuves diminuant de moitié).

Ces changements de comportements matrimoniaux se sont traduits par une transformation marquée de la structure des ménages et comme pour les tendances démographiques, on observe, en France, les mêmes évolutions que dans la plupart des pays industrialisés : augmentation des effectifs de personnes seules, de familles monoparentales et de couples non mariés ou sans enfant.

Figure Io. Évolution des ménages 1968-2009

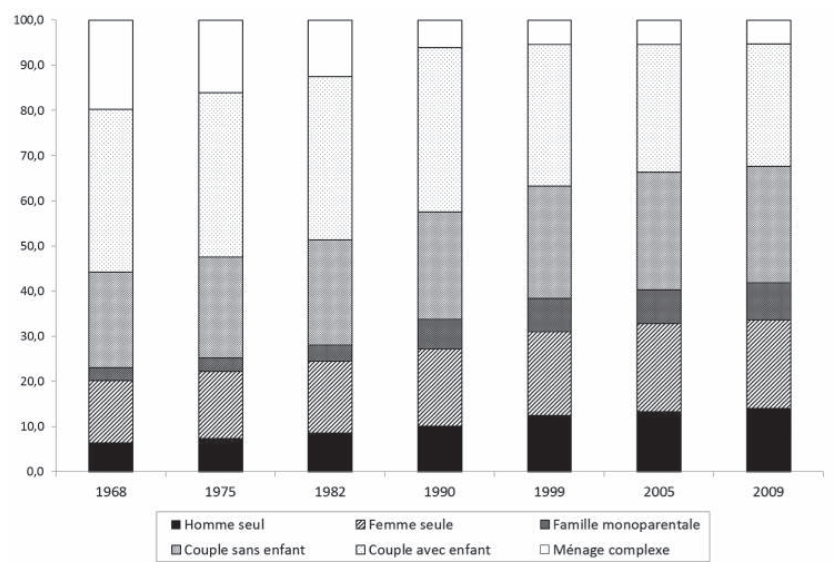

Source: Enquêtes Logement INSEE

La part des couples avec enfants dans l'ensemble des ménages est passée de $36 \%$ en 1968 à $27 \%$ en 2009 , tandis que celle des hommes seuls doublait (respectivement $7 \%$ et $14 \%$ ) et celle des familles monoparentales triplait presque (Figure Io). Or la part des familles monoparentales propriétaires dont la personne de référence est une femme âgée de 30 à 40 ans après avoir fluctuée autour de $20 \%$ entre 1984 et 2002 est redescendue en 2006 au niveau de 
1978, soit I4 \% (Figure I I). Par ailleurs, malgré une hausse des taux de propriétaires parmi les femmes divorcées âgées de plus de 60 ans de 1973 à 2006, les différences restent toujours très fortes ( $47 \%$ contre $86 \%$ pour les femmes mariées et $65 \%$ pour les veuves en 2006 - Figure I2).

Figure II. Évolution des taux de propriétaires selon le type de ménage dont la personne de référence est âgée de 30 à 40 ans

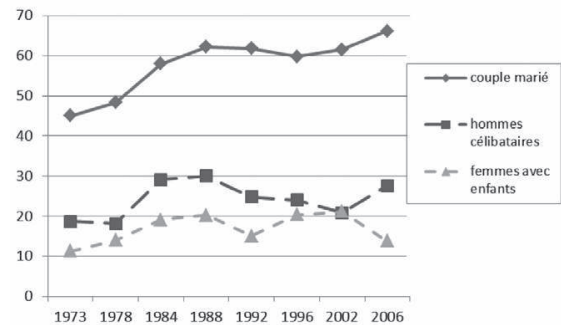

Figure I2. Évolution des taux de propriétaires des femmes veuves, divorcées et mariées lorsque la personne de référence a de plus de 60 ans

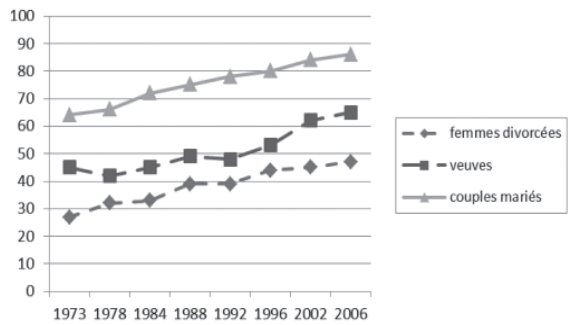

Source: Enquêtes Logement INSEE

Ainsi l'augmentation du nombre de ménages comprenant une personne seule avec ou sans enfants pourrait-elle expliquer en partie le tassement de la propriété de ces dernières années (Arbonville et Bonvalet, 2006). Un moyen simple de vérifier l'impact de ces changements sur le taux global de propriétaires consiste à appliquer au nombre de ménages à l'enquête logement de 2006 la structure matrimoniale par âge de 1978 et les taux de propriétaires observés par âge pour chaque type de ménage en 2006.

Ainsi en faisant l'hypothèse que les comportements matrimoniaux à chaque âge n'auraient pas changé entre 1978 et 2006, on peut calculer un taux global estimé de propriétaires $\mathrm{P}$ défini comme:

$$
P=\sum N_{i j}^{06}=\sum\left(M_{i j}^{06} X S_{i j}^{78} X T_{i j}^{06}\right)
$$

avec:

$\mathrm{N}_{\mathrm{ij}}^{06}$, nombre estimé de propriétaires par âge et type de ménage sous hypothèse d'invariance des comportements matrimoniaux par âge entre 1978 et 2006 ; $\mathrm{M}_{\mathrm{ij}}{ }^{06}$, nombre de ménages observés de type i dans la tranche d'âge j en 2006; 
$\mathrm{S}_{\mathrm{ij}}^{78}$, part du type de ménage j dans la tranche d'âge i en 1978 (structure matrimoniale par âge de 1978) ;

$\mathrm{T}_{\mathrm{ij}}^{06}$, taux de propriétaires observé dans la tranche d'âge i et le type de ménage j en 2006.

On obtient un taux de propriétaires supérieur à 60,2\% en 2006 contre $57 \%$ réellement observés.

Il est possible d'analyser la comparaison de ces taux observé et simulé, au cours du temps depuis l'enquête 1984 jusque l'enquête 2006. Le résultat présenté ci-dessous en figure i3 montre l'écart croissant, et traduit la part de plus en plus importante de nouveaux comportements matrimoniaux.

Figure I3. Taux de propriétaires observé et simulé

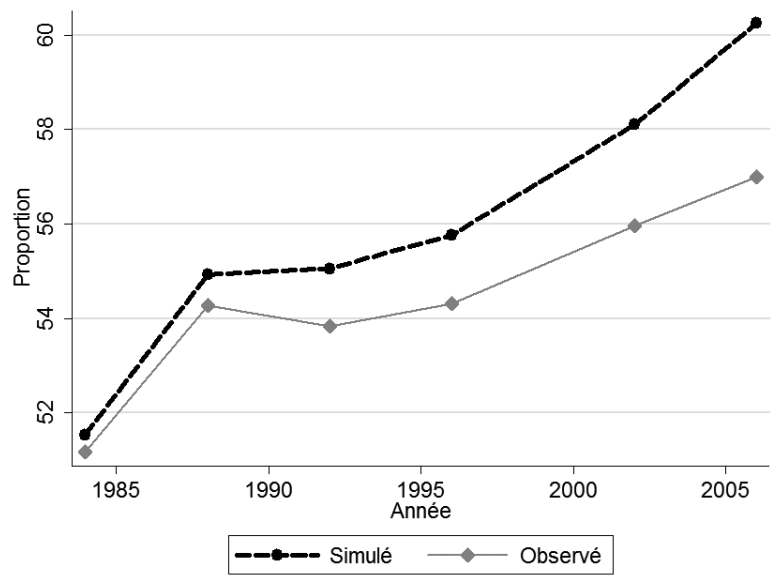

Enquêtes Logement 1984-2006, base 1978.

Le vieillissement de la population (en particulier le vieillissement des générations nombreuses du baby-boom) qui aurait dû se traduire par une hausse de la proportion de propriétaires en raison de l'effet cycle de vie (Arrondel, 2007) ${ }^{6}$ n’a donc pas suffi à contrecarrer le mouvement à la baisse lié aux transfor- 
mations de la famille et du couple amorcées par ces mêmes générations. Les politiques du logement favorisant l'accession à la propriété ont surtout profité aux couples qui avec deux salaires pouvaient s'endetter alors que les personnes seules avec ou sans enfant étaient plus fragiles.

\section{DES INÉGALITÉS ENTRE GÉNÉRATIONS ET CLASSES SOCIALES}

Plusieurs chercheurs ont cherché récemment à étudier à partir de modèles les inégalités intergénérationnelles d’accès à la propriété. Reprenant les enquêtes logement de 1973 à 1996, Daniel Courgeau et Monique Méron (2004) ont mis en évidence des différences d’accès à la propriété par génération et catégories sociales. Luc Arrondel (2007) s'appuyant sur les enquêtes patrimoine de l'INSEE et du panel européen a montré que, toutes choses égales par ailleurs, en particulier à niveau de patrimoine égal, les générations nées avant 1930 étaient plus souvent propriétaires que les générations nées après-guerre, notamment les générations nées entre 1950 et 1960. En revanche, pour Fanny Bugeja (2011), les générations nées entre 1930 et 1950 apparaissent, dans les enquêtes Budget de famille, les plus privilégiées, parce que dégagées des crédits d’achat. Enfin, considérant non pas les ménages mais l'ensemble des personnes propriétaires de leur logement, Marie-Émilie Clerc, Olivier Monso et Erwan Pouliquen (201 I) confirment l'avantage des générations nées entre 1940 et 1950, mais observent que les générations nées entre 1970 et 1975 connaissent une plus forte proportion de propriétaires entre 30 et 34 ans que les générations précédentes. Ainsi, selon les sources et entités (individus, ménages) retenues ainsi que les périodes étudiées, les résultats peuvent différer. Comme l'explique Luc Arrondel, il est toujours difficile de séparer les effets d'âge, de génération et de période dans la probabilité d'être propriétaire car «l'utilisation de données observées à un moment donné du temps pour inférer des effets longitudinaux ne permet pas de différencier les effets d'âge des effets de génération ou de période» (Arrondel, 2007, p. I84). 
C'est dans la lignée de ces travaux que nous nous situons en appliquant des modèles de régressions logistiques indépendants (un pour chaque Enquête Logement de 1978 à 2006) où nous calculons la probabilité d'être propriétaire selon certaines caractéristiques, identiques à chaque enquête, dans le choix des modalités prises en compte. Nous avons retenu comme Méron et Courgeau, la variable classes sociales (8 Postes), le type de ménage, l'âge du chef de ménage et le nombre d'enfants. Nous avons reconstruit dans le tableau i une variable recomposant l'âge du chef de ménage et le type de ménage, afin de tenir compte de l'effet d'interaction entre ces deux variables.

Le modèle confirme bien l'effet positif d'être en couple avec enfants sur la probabilité d'être propriétaire, quelle que soit la période considérée. À catégories sociales et CSP égales, les couples sans enfant ne sont plus souvent propriétaires que les couples avec enfants qu'à partir de 50 ans, c'est-à-dire après la décohabitation des enfants.

En ce qui concerne la classe sociale, les employés et ouvriers ont toujours eu, durant la période observée, une probabilité d’être propriétaire moins forte que les professions intermédiaires. En revanche, les cadres dont les chances de détenir leur logement ne se différenciaient pas ou peu de celles des professions intermédiaires jusqu'en 1996 voient leur probabilité d’être propriétaires significativement supérieure à ces derniers à partir de 2002. La hausse des prix immobiliers depuis dix ans, en dépit de la baisse des taux d'intérêt, a ainsi creusé l'écart entre les professions intermédiaires et les cadres (Bosvieux, 2005). En outre, la différence entre ouvriers et employés n’est jamais significative, traduisant une propension équivalente à être propriétaire à type de ménage fixé, ceci à chaque enquête Logement.

Nous avons ensuite calculé des probabilités issues de nouveaux modèles de régression logistique transversaux (un par année d'enquête), où l'âge et le type de famille ont été entrés séparément, et sous la forme d'une interaction. Cette interaction est significative, quelle que soit l'année d'enquête considérée $\left(\mathrm{p}<\mathrm{IO}^{-3}\right)$. Nous avons aussi détaillé dans ce nouveau modèle le nombre d'enfants du ménage en 4 classes (sans enfant, I à 2, 3 à 4, 5 et plus). 
Tableau I : Impact du type de ménage et de catégorie socio-professionnel sur le statut de propriétaire (logit binomial)

\begin{tabular}{|c|c|c|c|c|c|c|c|}
\hline & 2006 & 2002 & 1996 & 1992 & 1988 & 1984 & 1978 \\
\hline & ESTIMATEUR & ESTIMATEUR & ESTIMATEUR & ESTIMATEUR & ESTIMATEUR & ESTIMATEUR & ESTIMATEUR \\
\hline $\begin{array}{c}\text { TYPE } \\
\text { DE MÉNAGE }\end{array}$ & & & & & & & \\
\hline $\begin{array}{l}\text { COUPLE MOINS DE } \\
30 \text { ANS }\end{array}$ & $-1,957 * * *$ & $-1,865 * * * *$ & $-2,108 * * * *$ & $-2,060 * * *$ & $-1,775 * * * *$ & $-1,697 * * *$ & $-1,699 * * *$ \\
\hline $\begin{array}{l}\text { HOMME SEUL, } \\
\text { MOINS DE } 30 \text { ANS }\end{array}$ & $-3,264 * * *$ & $-3,259 * * *$ & $-3,126 * * * *$ & $-2,995 * * *$ & $-3,257 * * *$ & $-3,016 * * * *$ & $-3,279$ ******* \\
\hline $\begin{array}{l}\text { FEMME SEULE, } \\
\text { MOINS DE } 30 \text { ANS }\end{array}$ & $-3,467 * * * *$ & $-3,500 * * *$ & $-3,202 * * * *$ & $-3,443 * * *$ & $-3,232 * * *$ & $-2,947 * * *$ & $-2,909 * * *$ \\
\hline $\begin{array}{l}\text { HOMME SEUL, } 30 \\
\text { À } 49 \text { ANS }\end{array}$ & $-1,548 * * * *$ & $-|, 4| \mid * * * *$ & $-1,495 * * * *$ & $-1,446 * * * *$ & $-1,358 * * * *$ & $-1,253 * * * *$ & $-1,484 * * *$ \\
\hline $\begin{array}{l}\text { FEMME SEULE, } 30 \AA \\
49 \text { ANS }\end{array}$ & $-1,385 * * *$ & $-1,328 * * *$ & $-|, 4| 4 * * *$ & $-1,320 * * *$ & $-1,492 * * *$ & $-1,519 * * *$ & $-0,975 * * * *$ \\
\hline $\begin{array}{l}\text { COUPLE } 30 \AA \\
49 \text { ANS, AVEC } \\
\text { ENFANTS }\end{array}$ & ref. & ref. & ref. & ref. & ref. & ref. & ref. \\
\hline $\begin{array}{l}\text { COUPLE } 30 \AA \\
49 \text { ANS, SANS } \\
\text { ENFANTS }\end{array}$ & $-0,657 * * *$ & $-0,666 * * * *$ & $-0,58 \mid * * *$ & $-0,619 * * *$ & $-0,622 * * *$ & $-0,705^{*} * * *$ & $-0,278 * * *$ \\
\hline $\begin{array}{l}\text { COUPLE } 50 \text { ANS } \\
\text { ET PLUS, AVEC } \\
\text { ENFANTS }\end{array}$ & $0,320 * * * *$ & $0,311 * * * *$ & $0,369 * * *$ & $0,247 * * * *$ & $0,277 * * * *$ & $0,356 * * *$ & $0,328 * * * *$ \\
\hline $\begin{array}{l}\text { COUPLE } 50 \AA \\
69 \text { ANS, SANS } \\
\text { ENFANTS }\end{array}$ & $0,694 * * * *$ & $0,726 * * * *$ & $0,688 * * *$ & $0,648 * * * *$ & $0,573 * * * *$ & $0,504 * * *$ & $0,405 * * *$ \\
\hline $\begin{array}{l}\text { COUPLE } 70 \text { ANS } \\
\text { ET PLUS, SANS } \\
\text { ENFANTS }\end{array}$ & $0,819 * * *$ & $0,625 * * *$ & $0,285 * * *$ & $0,399 * * *$ & 0,134 & $0,272 * * *$ & $0,334 * * *$ \\
\hline $\begin{array}{l}\text { HOMME SEUL, } 50 \AA \\
69 \text { ANS }\end{array}$ & $-0,818 * * *$ & $-0,6655^{* * * *}$ & $-0,631 * * *$ & $-0,555 * * *$ & $-0,359 * * *$ & $-0,496 * * * *$ & $-0,417 * * *$ \\
\hline $\begin{array}{l}\text { FEMME SEULE, } 50 \AA \\
69 \text { ANS }\end{array}$ & $-0,612 * * * *$ & $-0,274 * * *$ & $-0,484 * * * *$ & $-0,448 * * * *$ & $-0,303 * * *$ & $-0,347 * * * *$ & $-0,484 * * * *$ \\
\hline $\begin{array}{l}\text { HOMME SEUL, } 70 \\
\text { ANS ET PLUS }\end{array}$ & $-0,138$ & $-0,342 * * *$ & $-0,680 * * *$ & $-0,403 * * *$ & $-0,47 \mid * * *$ & $-0,296 *$ & $-0,4 \mid 2 * *$ \\
\hline $\begin{array}{l}\text { FEMME SEULE, } 70 \\
\text { ANS ET PLUS }\end{array}$ & $-0,354 * * * *$ & $-0,454$ ****** & $-0,637 * * *$ & $-0,879 * * * *$ & $-0,8 \mid 8 * * *$ & $-0,704 * * * *$ & $-0,55 \mid$ ***** \\
\hline $\begin{array}{l}\text { HOMME SEUL AVEC } \\
\text { ENFANT, } 30 \AA \\
49 \text { ANS }\end{array}$ & $-1,015 * * *$ & $-0,649 * * *$ & $-0,586 * *$ & $-1,002 * * *$ & $-0,951 * * * *$ & $-0,583 * *$ & $-0,08$ \\
\hline
\end{tabular}




\begin{tabular}{|c|c|c|c|c|c|c|c|}
\hline $\begin{array}{l}\text { FEMME SEULE AVEC } \\
\text { ENFANT, } 30 \AA \\
49 \text { ANS }\end{array}$ & $-1,57 \mid * * * *$ & $-1,396 * * *$ & $-1,356 * * * *$ & $-1,386 * * *$ & $-1,249 * * * *$ & $-1,188 * * *$ & $-0,978 * * * *$ \\
\hline $\begin{array}{l}\text { HOMME SEUL AVEC } \\
\text { ENFANT, } 50 \text { ANS } \\
\text { ET + }\end{array}$ & $-0,545 * *$ & $-0,142$ & $-0,068$ & $-0,024$ & $-0,363$ & $-0,374$ & $-0,236$ \\
\hline $\begin{array}{l}\text { FEMME SEULE AVEC } \\
\text { ENFANT, } 50 \text { ANS } \\
\text { ET + }\end{array}$ & $-0,497 * * *$ & $-0,383 * * *$ & $-0,294 * *$ & $-0,17$ & $-0,417 *$ & $-0,215$ & $-0,207$ \\
\hline AUTRES & $-1,032 * * * *$ & $-1,076 * * *$ & $-0,934 * * * *$ & $-1,223 * * * *$ & $-0,834 * * * *$ & $-0,582 * * * *$ & $-0,359 * * * *$ \\
\hline $\begin{array}{l}\text { CATÉGORIE } \\
\text { SOCIO } \\
\text { PROFESSION- } \\
\text { NELLE }\end{array}$ & & & & & & & \\
\hline AGRICULTEUR & $1,009 * * * *$ & $0,688 * * * *$ & $0,558 * * * *$ & $0,760 * * *$ & $0,488 * * * *$ & $0,494 * * *$ & $0,798 * * * *$ \\
\hline ARTISAN & 0,07 & $0,220 * *$ & $0,175 *$ & $0,176 * *$ & $0,208 * *$ & $0,152 *$ & $0,286 * * * *$ \\
\hline CADRE & $0,272 * * *$ & $0,236 * * * *$ & $0,114 *$ & $-0,024$ & 0,042 & $-0,07$ & $0,134 *$ \\
\hline $\begin{array}{l}\text { PROFESSION } \\
\text { INTERMÉDIAIRE }\end{array}$ & ref. & ref. & ref. & ref. & ref. & ref. & ref. \\
\hline EMPLOYÉS & $-0,818 * * *$ & $-0,689 * * *$ & $-0,586 * * * *$ & $-0,568 * * *$ & $-0,495 * * *$ & $-0,572 * * *$ & $-0,299 * * *$ \\
\hline OUVRIER & $-0,800 * * * *$ & $-0,622 * * *$ & $-0,546 * * *$ & $-0,533^{*} * *$ & $-0,458 * * *$ & $-0,554 * * *$ & $-0,358 * * *$ \\
\hline RETRAITÉ & 0 & $0,123 *$ & $0,137 *$ & $-0,038$ & $-0,075$ & $-0,136 *$ & 0,058 \\
\hline SANS ACTIVITÉ & $-0,890$ **** & $-0,676 * * *$ & $-0,593 * * * *$ & $-0,460$ **** & $-0,446$ **** & $-0,504 * * *$ & $-0,144$ \\
\hline CONSTANTE & $0.960 * * *$ & $0.798 * * *$ & $0.735 * * *$ & $0.787^{* * * *}$ & $0.728 * * *$ & $0.617^{* * *}$ & $0.189 * * *$ \\
\hline $\mathrm{N}$ & 42963 & 32156 & 29043 & 36222 & 26686 & 29233 & 23492 \\
\hline
\end{tabular}

$* p<0.05$, *** $p<0.01$, ***** $p<0.001$

La figure 14 représente le résultat de ces probabilités estimées à certains âges aux différentes enquêtes. On remarque notamment que pour les couples de I à 2 enfants, dont la personne de référence est âgée de 30 à 34 ans, et les femmes de 30 à 34 ans seules avec enfant, l'écart entre cadres / professions intermédiaires et employés / ouvriers augmente continument depuis 1992, et particulièrement depuis 2002. Ainsi, les employés et ouvriers accèdent-ils de plus en plus difficilement à la propriété, malgré l'allongement de la durée d'endettement et la baisse des taux d'intérêt, seules les classes moyennes et surtout les classes supérieurs pouvant faire face à la hausse des prix immobiliers depuis le début du siècle. 
Ces inégalités se retrouvent à la veille de la retraite parmi les ménages dont la personne de référence est âgée de 55 à 59 ans, de façon moindre chez les couples sans enfant $(87 \%$ pour les cadres et $70 \%$ pour les ouvriers en 2006 , contre $67 \%$ et $55 \%$ en 1978 ) et accentuée chez les femmes seules de cet âge (68\% de propriétaires parmi les cadres et 4I \% parmi les ouvriers en 2006 contre 49 et $37 \%$ en I978).

Figure I4: Probabilités estimées d'être propriétaire selon le type de ménage et l'âge de la personne de référence

Couples avec I ou 2 enfants 30-34 ans

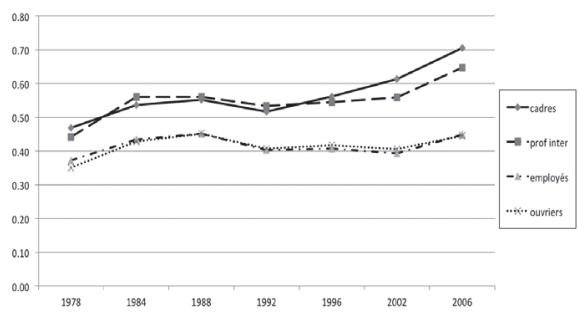

Couples avec I ou 2 enfants 35-39 ans

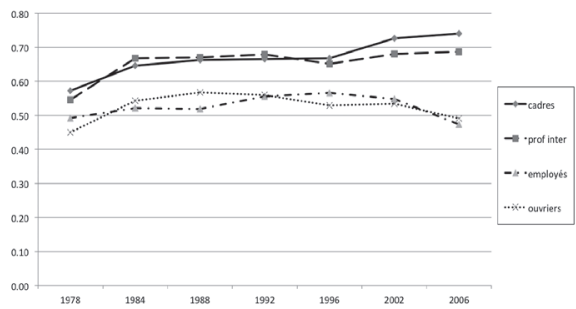

Couples sans enfant de 55 à 59 ans

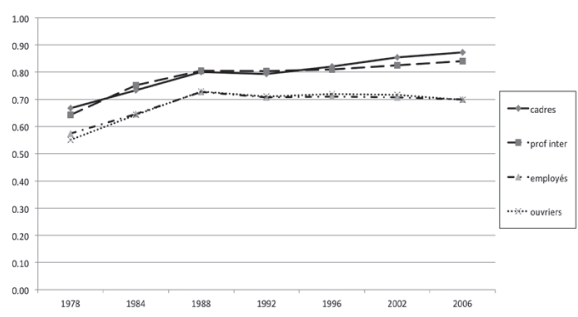

Femmes seules de 30-34 ans avec enfants

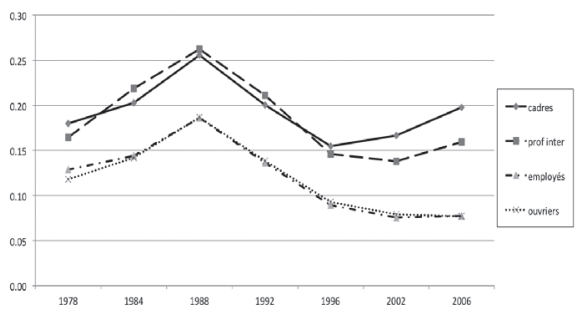

Femmes seules de 35 à 39 ans avec enfants

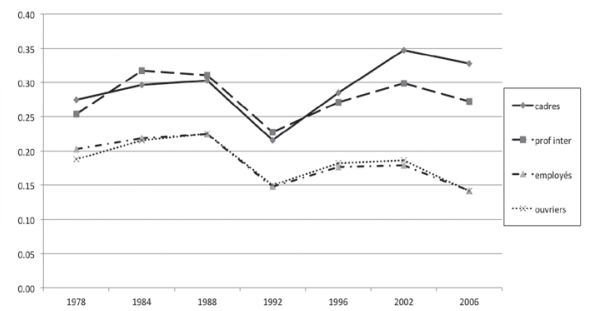

Femmes seules de 55 à 59 ans

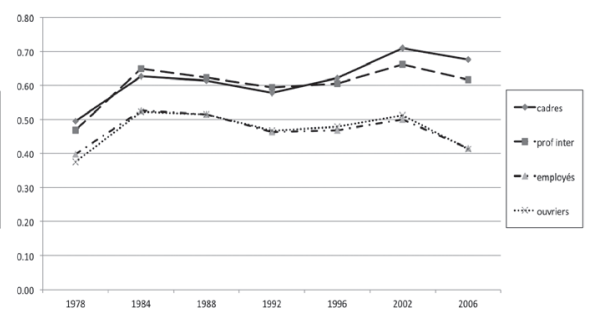




\section{CONCLUSION}

Depuis le milieu du $\mathrm{Xx}^{\mathrm{e}}$ siècle, les politiques du logement ont cherché à résoudre les problèmes de logement en favorisant l'accès à la propriété des ménages. Les réformes du crédit bancaire et les lois incitant à l’accession à la propriété (en particulier celle de 1977) ont surtout profité aux couples qui ont pu s'endetter, notamment grâce à l'apport du salaire de la femme. Cependant toutes les classes sociales nont pas bénéficié de la même manière des réformes. Au cours de la période 1978 et 2006, si l'écart se creuse entre les classes supérieures ou moyennes et les ouvriers ou employés, les inégalités sociales apparaissent encore plus fortes parmi les femmes seules avec ou sans enfant que parmi les couples, révélant les limites d'une politique du logement centrée uniquement sur l'accession à la propriété des couples et laissant ainsi de côté toute une partie de la population. En effet, les évolutions de la famille avec l'augmentation des divorces et séparations ont généré de nouveaux parcours résidentiels initiés par les premières générations du baby-boom avec un retour vers le locatif après une accession à la propriété. Elles expliquent en partie le ralentissement de la hausse des taux de propriétaires au cours des deux dernières décennies.

NDA. Une version précédente de cet article a fait l'objet d'une communication au $17^{\mathrm{e}}$ colloque international de l'AIDELF, «Démographie et Politiques Sociales», qui s'est tenu à Ouagadougou (Burkina Faso) du 12 au 16 novembre 2012. 


\section{BIBLIOGRAPHIE}

ARBONVILLE D., 2006, «Évaluer les implications des évolutions sociodémographiques sur l'habitat en Europe», in Famille, habitat et urbanisation en Europe du sud, «Cahiers de l'INED» Paris, INED, nI57, p. 4I-66.

-, BONVALET C., 2006, «Famille, logement et urbanisation en France», in Famille, habitat et urbanisation en Europe du sud, «Cahiers de l'INED» Paris, INED, nI57, p. 237-274.

ARRONDEL I., 2007, «Patrimoine logement et cycle de vie», in Vieillissement de la population et logement: les stratégies résidentielles et patrimoniales, C. Bonvalet et al. (éds), Paris, La Documentation Française.

BUGEJA F., 20II, «Les inégalités d'accès à la propriété et leurs déterminants institutionnels. Étude comparative entre la France et le Royaume-Uni (1980-2005)», Revue Française de Sociologie, 52, p. 37-69.

BONVALET C. BRINGÉ A., 1988, Cycle de vie et changements urbains en Région parisienne: histoire résidentielle, Rapport de recherche INED, CNAF, DREIF, MELATT.

-, CLÉMENT C. OGG J., 20II, Réinventer la famille. L'histoire des baby-boomers, Paris, PUF, «Le lien social».

BOSVIEUX J., 2005, «Accession à la propriété: des acquéreurs plus nombreux mais prudents», Économie et Statistique, 38I-382, p. 41-61.

BOURDIEU P., 2000, Les Structures sociales de l'économie, Paris, Seuil.

CHAUVEL I., 1998, Le Destin des générations. Structure sociale et cohortes en France au $X x^{e}$ siècle, Paris, PUF, «Le lien social ».

CLERC M.E, MONSO O., POULIQUEN E., 20II, «Les inégalités entre générations depuis le baby-boom, l'économie française», in L'Économie française, «Insee Références», Paris, INSEE, p. 46-67.

CUTURELLO P. (éd.), 1992, Regard sur le logement: une étrange marchandise, Paris, L'Harmattan.

DRIANT J-C., 2009, Les Politiques du logement en France, Paris, La Documentation française. 
JOSEPH I., 1976, «Tactiques et figures disciplinaires», in Disciplines à domicile: édifıcation de la famille, éd. I. Joseph, P. Fritsch, A. Battegay, Recherches n²8, p. 29-208.

GOTMAN A., 1986, Transmission du patrimoine et trajectoires familiales, Paris, IPRAUS-CNAF.

GROUX G., LÉVY C., 1993, La Possession ouvrière: du taudis à la propriété (XIXe-XXe siècles), Paris, Éditions de l'Atelier.

LACAZE J-P., 1997, Les Politiques du logement, Paris, Flammarion, «Dominos ».

LAFERRÈRE A., 2007, «le logement dans l'accumulation du patrimoine des personnes âgées », in Vieillissement de la population et logement: les stratégies résidentielles et patrimoniales, C. Bonvalet et al. (éds), Paris, La Documentation française, p. 149-162.

LEFEBVRE B., MOUILLART M., OCCHIPINTI S., 1992, Politique du logement: 50 ans pour un échec, Paris, L'Harmattan, Paris.

LEVY J-P., 2007, «Les 50 ans et plus dans la hiérarchisation sociale du parc immobilier français (1978-2002): un rôle prépondérant»», in Vieillissement de la population et logement: les stratégies résidentielles et patrimoniales, C. Bonvalet et al. (éds), Paris, La Documentation française, p. 267-28l.

LOISEAU M., BONVALET C., 2005, «L'impact de loi de 1948 sur les trajectoires résidentielles en Île-de-France», Population, 60-3, p. 351-366.

LOUVOT-RUNAVOT C., 200I, «Le logement dans I'Union européenne: la propriété prend le pas sur la location», Économie et Statistique, 343, p. 29-50.

MAISON D., 1993, «Effet d'alliance et transmission différée dans le rapport à la propriété et à l'habitat» in Le Logement une affaire de famille, Paris, L'Harmattan, p. 87-109.

MERLIN P.,1988, «L'évolution du parc de logements (1945-1986)», in Transformation de la famille et Habitat, C. Bonvalet, P. Merlin (éds), INED, «Travaux et Documents», 120, p.771-783.

MERON M., COURGEAU D., 2004, «Home ownership and social inequality in France », in Home ownership and social inequality in comparative perspective, K. Kurtz, H.-P. Blossfeld (eds), Stanford, Stanford University Press, p. 6I-78. 
RAPPORT NIOL, 1985, L'Évolution des structures familiales et ses conséquences sur l'habitat, Rapport de la Commission nationale de l'Habitat.

RAYMOND H. RAYMOND M-G., HAUMONT N, 1966, L'Habitat pavillonnaire, Paris, CRU.

SARMA C., 1985, «La cohabitation hors mariage: un mode de vie différent?» Économie et Statistique, 179, p.47-52.

SAUVY A., 1965, Histoire économique de la France entre-les-deux guerres, Paris, Fayard.

TAFFIN C., 1987, «L'accession à tout prix», Économie et Statistique, 202, p. 5- 15.

TOPALOV C., 1987, Le Logement en France. Histoire d'une marchandise impossible, Paris, Presses de la Fondation Nationale des Sciences Politiques. 
